Gallardo Fernández, I.M. \& Saiz Fernández, H. (2016). Emociones y actos comunicativos desde la dramatización de situaciones cotidianas. Una propuesta de intervención en Educación Primaria. Revista Electrónica Interuniversitaria de Formación del Profesorado, 19(3), 219-229.

DOI: http://dx.doi.org/10.6018/reifop.19.3.267361

\title{
Emociones y actos comunicativos desde la dramatización de situaciones cotidianas. Una propuesta de intervención en Educación Primaria
}

Isabel M. Gallardo Fernández, Héctor Saiz Fernández

Universitat de Valencia

\section{Resumen}

La realidad socioeducativa actual demanda de los profesionales de la educación una intervención comprometida y coherente para el desarrollo de competencias y habilidades emocionales. Es necesario complementar lo académico y cuantificable con aquello que sobrepasa las barreras del centro e influye en el rendimiento y desarrollo personal y social del alumnado. Las relaciones que se dan entre los componentes de la triada emoción, pensamiento y acción regulan cada momento de nuestros días, pudiéndose ver estas alteradas negativamente por estímulos externos que en ocasiones, no somos capaces de gestionar adecuadamente. Esta incorrecta gestión emocional tiene su base en un creciente analfabetismo emocional que nos lleva a respuestas impulsivas y no reflexivas.

Este artículo plantea una propuesta de intervención en el aula de Educación Primaria que tiene como base el diálogo y la cooperación para el desarrollo de competencias-habilidades emocionales desde el acto comunicativo-dramático, enfatizando en los beneficios que esta herramienta pedagógica tiene para con el desarrollo de espacios inclusivos donde los estudiantes puedan expresarse libremente. Esta propuesta tiene un carácter abierto y su concreción vendrá determinada por el contexto y la implementación en las aulas. El trabajo concluye planteando la conveniencia de la puesta en práctica de Programas de Educación Emocional.

\section{Palabras clave}

Educación de la afectividad; dramatización; comunicación interpersonal; contexto de aprendizaje. 


\title{
Emotions and communicative acts from the dramatization of everyday situations. An educational intervention proposal in Primary Education
}

\begin{abstract}
Nowadays the socio-educational context demands from teachers a committed and coherent intervention to promote emotional skills and competences. It is necessary to complement the academic and quantifiable dimension with other knowledge that has overcome school barriers and has an important role in the capacities and development of students. The relations between the triad: emotion, thought and action, control every moment of our daily life. This trio can be disturbed by external stimuli, which sometimes cannot be solved in a positive way. This mismanagement is due to the increasing "emotional illiteracy" that makes us act in an impulsive and thoughtless manner.

This paper shows an educational proposal to contribute to the development of competences and skills in the Primary Education classroom, based on dialogue, cooperation and dramatic act as learning tools, emphasizing the benefits that this resource has to develop inclusive spaces where students can express themselves freely. This project has an open character, its realisation will be determined by the context and its implementation in the classroom. This paper concludes the convenience of emotional projects and their implementation in educational institutions.
\end{abstract}

\section{Key words}

Affective education; dramatization; interpersonal communication; learning environment.

\section{Introducción}

A lo largo de los años la educación se ha orientado hacia la producción, gestión y transmisión de información, considerando el proceso educativo como un camino hacia el éxito profesional y laboral futuro. Hoy en día, comprobamos como esa concepción academicista de la "educación exitosa" no tiene los resultados positivos esperados. Cada vez más, se hace evidente la necesidad de reformular el hecho educativo, de replantear nuestros objetivos en relación con el contexto variable y complejo que vivimos. Se trata de concebir la educación desde una perspectiva más humanista centrada en el respeto a la vida, la dignidad humana y el bienestar de las personas (UNESCO, 2015).

Es este sentido, la educación emocional es una dimensión que ha sido desatendida tradicionalmente en los contextos educativos. Afortunadamente, hoy somos más conscientes de su influencia en el proceso de aprendizaje y en los procesos de desarrollo y contribución al bienestar personal y social. Como expone Bisquerra (2012) existe una relación significativa entre emociones y exclusión-inclusión, en tanto que las emociones estimulan comportamientos impulsivos, los cuales si son mal gestionados, desembocan en manifestaciones relacionadas con la ira (enfado, rabia, odio, etc.), tan habituales e identificables hoy en día en nuestras comunidades y entre los más jóvenes.

Vivimos pues en una sociedad cada vez más caracterizada por un creciente analfabetismo emocional. Los resultados a nivel social son evidentes: aumento de actitudes individualistas, poco solidarias, egoístas e incluso violentas. Las manifestaciones entre los jóvenes en edad 
escolar son muy variadas: depresión, ansiedad, consumo de sustancias adictivas, abusos, soledad, aislamiento, desórdenes alimenticios, incluso suicidio, entre otras muchas. El sistema educativo que camina hacia el horizonte de la excelencia académica cuantificable, sin embargo, en ocasiones desatiende la dimensión emocional y más personal del discente.

Para nosotros, las necesidades prioritarias del alumnado de Educación Primaria están vinculadas con lo que viven en su realidad y contexto familiar, social y cultural. Asumimos que la sociedad en general se ha orientado hacia la búsqueda de resultados evidentes, inmediatos y cuantificables, olvidándonos a veces, que además de seres racionales somos emocionales, capaces de sentir una variedad enorme de emociones diferentes sin que en muchos casos seamos conscientes de ello. Las emociones determinan nuestras decisiones y hasta conducen nuestras vidas. Los docentes hemos de asumir la complejidad de las interacciones que se dan en los procesos educativos más allá de los puramente académicos y cuantificables.

La escuela ha de tener presente que ante todo somos personas vivas y diversas, seres emocionales capaces de sentir y pensar, y sobre todo, capaces de "sentipensar". Ante la realidad social que vivimos es el momento de un cambio profundo y de calidad en los procesos educativos, marcado por el cultivo de la emoción y las prácticas educativas contextualizadas en la realidad. Dejemos de ocultar y negar nuestros sentimientos, aprendamos a gestionar las emociones trabajando sobre ellas, para un mayor bienestar personal y social de la comunidad educativa y de la población en general.

El alumno como pleno sujeto de derechos es un ser complejo, continuamente influido por factores emocionales a los que los docentes hemos de responder desde el contexto educativo para facilitar su desarrollo integral. Por ello, es necesario considerar las emociones dentro del proceso de enseñanza-aprendizaje. De acuerdo con este planteamiento señalamos los beneficios y posibilidades que ofrece el acto dramático como instrumento para desarrollar habilidades, educar en valores, favorecer conocimientos y conductas positivas desde el enfoque del conjunto de competencias emocionales (CE) que recoge Bisquerra y Pérez (2007).

En esta primera parte del capítulo nos centramos en justificar la importancia de incluir la educación emocional en los procesos educativos escolares y en fundamentar su relevancia desde las teorías psicoeducativas y de la comunicación más destacadas. Pretendemos así señalar la necesidad de considerar las emociones como parte sustancial del proceso de enseñanza aprendizaje en la escuela del siglo XXI. En la segunda parte, introducimos lo que supone el acto dramático como herramienta pedagógica relacionado con la educación emocional, enfatizando en sus beneficios y posibilidades en el contexto escolar. En este punto, planteamos una propuesta de intervención en el aula de Educación Primaria que construimos con la intención de que resulte útil y provoque la reflexión en diferentes realidades educativas. Se trata de una propuesta abierta que permite la interpretación personal. Huimos del impulso de generalizar acciones en educación puesto que pensamos que esto tiene resultados cuanto menos inciertos.

\section{Enfoque y objetivos del acto educativo emocional}

Previamente al desarrollo de la fundamentación y con la finalidad de acercar los contenidos de este texto al contexto educativo, exponemos a continuación unas consideraciones necesarias en relación con los requisitos y sentido de las acciones y procesos de educación emocional. 
En primer lugar, hay que resaltar que todo proyecto, acción e intervención a nivel educativo conllevará unos objetivos que deberán construirse de forma concreta, reduciendo lo más posible su ambigüedad. En nuestro caso, planteamos cuatro objetivos generales elaborados desde el planteamiento de intervención a través del acto dramático. Los presentamos a continuación a modo de ejemplo: a) favorecer una actitud positiva ante la vida desarrollando en el alumnado habilidades y estrategias de gestión emocional, b) educar en la gestión de emociones propias/ajenas a través del desarrollo de las CE, C) potenciar la creatividad, su desarrollo afectivo-emocional y capacidad expresiva en una realidad compleja y d) aprender a afrontar situaciones conflictivas que surgen del choque entre diferentes reacciones emocionales. Estos objetivos adquirirán mayor concreción cuando se formulen para un determinado contexto educativo.

En segundo lugar, hemos de señalar la importancia de integrar la acción de educación emocional, ya sea proyecto o actividad, en la cultura de todo el centro. Es decir, de nada sirve reproducir mecánicamente una actividad de educación emocional si no se reflexiona sobre ello y se comparte su sentido. Los proyectos, y de forma más destacada los proyectos emocionales, han de impregnar la vida del centro, compartirse y que exista una implicación profunda de todos los agentes, que crean en el proyecto, en sí mismos y en los estudiantes (tarea que requiere un trabajo de coordinación más complejo a nivel de aula y de centro). Habremos de conseguir contagiar nuestro proyecto a toda la comunidad educativa y hacer que nuestra aula sea el espacio donde se respire, en este caso, la emocionalidad de las personas.

Por último, destacar la importancia de atender al individuo desde un enfoque holístico. Respondiendo de este modo ante las directrices de normativas estatales e internacionales en materia educativa y contribuyendo al desarrollo integral del sujeto en conexión con los diferentes contextos en los que nos desarrollamos. Se favorece así el desarrollo del sujeto en un ambiente de comunidad, donde los valores de solidaridad, compromiso, sostenibilidad y respeto adquieren un mayor significado.

\section{El poder comunicativo de las emociones}

Las emociones han sido a lo largo de la historia las que han posibilitado la permanencia en la tierra del ser humano, resolviendo satisfactoriamente determinadas decisiones que ponían en peligro la continuidad de la especie. En su proceso evolutivo, el hombre ha llegado a codificar parte del lenguaje emocional para constituir un código de comunicación que, a menudo, conlleva respuestas y disposiciones emocionales involuntarias de quienes nos rodean.

Estudiosos reconocidos en el tema señalan que las respuestas emocionales tienen dos acepciones: una interna, que experimenta el individuo y que le resulta difícil de expresar (componente subjetivo), y otra externa con carácter conductual (respuesta vegetativa y motora), cuyo código es comprendido y compartido por los miembros de la comunidad (Mora, 2013). La interpretación de ese código emocional compartido se hace al dotar de significado determinadas respuestas fisiológicas involuntarias representadas a través de la comunicación no verbal (Rulicki \& Martín, 2012). Es la carga emocional de las respuestas humanas la que se relaciona directamente con el lenguaje no verbal y con el acto real de comunicar. Esta traducción fisiológica de las emociones conforma un lenguaje global con millones de interpretaciones diferentes, siendo el lenguaje de las emociones parte esencial de nuestra capacidad comunicativa. 
En este sentido, el área de Lengua y Literatura es una de las áreas curriculares del sistema educativo cuyo carácter instrumental resulta esencial para que se produzcan situaciones de aprendizaje en el resto de áreas de conocimiento. El desarrollo de las habilidades comunicativas, verbales y no verbales, es imprescindible para el desenvolvimiento futuro del alumno en el mundo y para la estructuración de su pensamiento (Bruner, 1998).

Según Gómez (2003), el lenguaje es el instrumento común de comunicación por el que modificamos la realidad que nos rodea, siendo cada vez más importante el estudio de los conocimientos comunicativos que van más allá del código lingüístico (cómo decirlo, qué decir y a quién dirigirse), como expone Prado (2011) al referirse a la etnografía de la comunicación. Es en la habilidad para influir en la realidad donde entran en juego las emociones y su poder comunicativo.

Para reforzar la importancia del acto comunicativo como instrumento de aprendizaje emocional, nos remitimos a la obra de Paulo Freire (1997), donde expone que la educación bancaria ha de ser sustituida por una escuela donde la comunicación y el diálogo sean la base del aprendizaje. Para ello, el discurso del educador debe partir de la situación en la que el alumno se encuentra y tratar de trasformar esa realidad siempre desde el respeto personal. El diálogo entre profesor y aprendiz será la base para el desarrollo de los aprendizajes y de la inteligencia emocional.

\section{El acto dramático como recurso pedagógiico}

El drama constituye un laboratorio de la realidad o un ensayo para la vida (Mato, 2006). Nos permite acercar diferentes realidades al contexto educativo e interactuar en ellas sin que esto implique consecuencias directas para los participantes, como ocurriría en la vida real (Mallika, 2000). Los procesos educativos con base en el acto dramático facilitarán un aprendizaje vivencial a través de simulaciones en contexto imaginados y planificados, en los que la implicación de nuestras emociones, afectividad e imaginación es la base de su construcción (Winston, 2000; Zillmann, 1994). El acto dramático nos permite explotar nuestras potencialidades como comunicadores y también permite el análisis reflexivo posterior sobre lo acontecido en el proceso.

Además, el acto dramático construye un espacio donde pueden desarrollarse procesos de educación en valores, los cuales suelen ser difícilmente concretados en los contextos educativos (Núñez y Navarro, 2007). Dicha educación en valores no es objeto de teorización ni planteamientos academicistas, debe construirse en la alteridad y desde la relación con el otro, condición que nos facilita el espacio relacional del acto dramático.

A pesar de los beneficios y las posibilidades que aporta el acto dramático como recurso pedagógico, la escasa formación de los profesionales de la educación en esta estrategia hace que no esté presente por lo general en nuestras aulas (Navarro Solano, 2007). Es importante aclarar que el acto dramático no es lo mismo que el acto teatral. Según Pérez (2004), el teatro se centra en desarrollar capacidades estéticas del individuo, mientras que la dramatización conlleva una resolución de problemas a través de la experiencia simulada de situaciones comunes.

Como señalábamos anteriormente, el lenguaje de las emociones es parte esencial de nuestra capacidad comunicativa y determina significativamente la intención y respuesta recibida. Por esto y por los beneficios que hemos ido analizando sobre el acto dramático, consideramos al mismo como instrumento práctico de gran valor pedagógico donde confluyen emociones y comunicación (Pérez, 2004). Es por ello por lo que nos planteamos 
fundamentar nuestra Propuesta en el uso del acto dramático como recurso pedagógico dentro de un proceso de educación emocional enfocado al alumnado de Educación Primaria. La dramatización de situaciones cotidianas conflictivas, en las que la carga emocional sea significativa y su gestión no sea adecuada, posibilitará el trabajo de educación emocional en los grupos de CE que Bisquerra (2003) analiza en su obra "Educación emocional y competencias básicas para la vida".

\section{Propuesta de intervención en Educación Primaria: educar emocionalmente desde la dramatización de situaciones cotidianas}

Las emociones impregnan cada momento de nuestras vidas y de nuestra cotidianidad. El saber gestionarlas adecuadamente nos permitirá afrontar nuestro día a día más eficientemente. Emoción, pensamiento y acción son los tres pilares que hilan cada instante de nuestro ser, de ahí la importancia de ahondar en ese tipo de conocimiento para afrontar determinadas situaciones y desenvolvernos plenamente en nuestra sociedad. El proceso educativo emocional es continuo y permanente a lo largo de la trayectoria vital de las personas, es parte esencial de los componentes que garantizan un desarrollo integral de la persona estrechamente relacionado con su bienestar personal y social.

La propuesta que presentamos a continuación se enmarca en el Curriculum de Primaria, en el área de Lengua Castellana y Literatura, por su relación directa con la competencia comunicativa y elementos extra-verbales. Además, viene fundamentada por las directrices del Real Decreto 126/2014, de 28 de febrero, por el que se establece el currículo básico de la Educación Primaria y que define el planteamiento de aprendizaje por competencias como la "combinación de habilidades prácticas, conocimientos, motivación, valores éticos, actitudes, emociones, y otros componentes sociales y de comportamiento que se movilizan conjuntamente para lograr una acción eficaz” (Pág. 2).

Asumimos con Bisquerra (2003:22) que "las competencias emocionales son el conjunto de conocimientos, capacidades, habilidades y actitudes necesarias para comprender, expresar y regular de forma apropiada los fenómenos emocionales". Desde el trabajo de cinco grupos de CE el alumnado desarrollará, entre otras, capacidades de reconocimiento de sentimientos propios/ajenos, así como recursos y estrategias para identificar señales orgánicas que preceden respuestas emocionales a menudo involuntarias (como el caso de la tensión muscular y rubor que preceden una respuesta intensa en procesos de enfado). En este sentido, resulta necesario reflexionar sobre las señales orgánicas, emociones generadas, su influencia en el comportamiento, la gestión del mismo y su éxito/fracaso en el contexto educativo, y eso lo potenciaremos mediante la dramatización de escenas cotidianas.

Basándonos en la teoría sobre educación emocional de Bisquerra y en el acto dramático, presentamos como propuesta la elaboración colaborativa de un conjunto de escenas de dramatización que constituirán un espacio de desenvolvimiento libre donde los estudiantes pueden expresarse en el contexto de la escena representada. Es importante posibilitar que en el proceso educativo los alumnos participen activamente, por ello consideramos que la creación de las escenas es un momento clave del proceso, además de la propia dramatización. Es también en el proceso de "creación dialógico" donde se van a poner en juego un conjunto amplio de habilidades, actitudes y competencias individuales-grupales que favorecerán el aprendizaje significativo.

\section{Secuencia de la Propuesta}

Toda actuación pedagógica precisa de una planificación previa, una secuenciación necesaria que guíe el proceso pero que no limite la capacidad de improvisación ni destruya la 
sorpresa, dos elementos que gestionados adecuadamente son facilitadores del aprendizaje y potencialmente motivadores. En la Propuesta que planteamos diferenciamos cuatro etapas que servirán de marco de referencia para repensar la planificación en un contexto determinado. A continuación, presentamos cuatro esquemas de contenidos que se corresponden con las cuatro fases del proceso y que facilitarán la comprensión del mismo.

\section{Fase de planificación}

1.1 Concreción de la Propuesta, adaptación al contexto y características de los participantes

- Concreción del proyecto, unidad didáctica, actividad o taller.

- Aspectos metodológicos (formulación de objetivos, contenidos, interés y motivación del alumno, gestión de espacios, integración en unidades didácticas, medios y recursos, etc.).

- Planificación de tareas, actividades y acciones a realizar.

- Temporalización (concreción de sesiones, transiciones entre fases del Proyecto para una progresión coherente del proceso).

- Evaluación durante el proceso y estrategias de evaluación conjuntas.

1.1.1 En este punto, los cinco grupos de competencias emocionales que plantean Bisquerra y Pérez (2007) facilitarán la labor de planificación docente. Estos constituyen conjuntos de competencias emocionales que servirán para contextualizar las actividades y tareas, así como para situar el tipo de conflicto que se desarrollará en las escenas sobre las que se trabaje. Los grupos son los siguientes:

1 Conciencia emocional.

2 Regulación emocional.

3 Autonomía emocional.

4 Competencia social.

5 Competencias para la vida y el bienestar.

1.2 Presentación e introducción de la propuesta de Educación Emocional

- Implicación de todos los participantes.

- Serán garantía de éxito el entusiasmo, compromiso e implicación lograda.

\section{Fase de creación de las escenas}

2.1 Planteamiento conjunto de situaciones conflictivas cercanas a los estudiantes

- Ejemplos de una incorrecta gestión emocional: enfados, rabietas, faltas de respeto, actitudes egoístas, abusos, entre otras.

- Estrategias facilitadoras: grupos de discusión, debates a partir de historias, uso de noticias en prensa, recopilación previa de situaciones de otras sesiones, etc.

2.2 Organización del alumnado en grupos. Selección de las escenas a representar

- Composición de grupos heterogéneos. 
- Proceso de negociación entre los integrantes para la selección de una la escena sobre la que trabajarán.

\subsection{Construcción de la escena}

- Elaboración guiada y estructurada del guion para el tiempo acordado (personajes, título y trama con introducción, desarrollo, clímax y desenlace). En este punto es importante remarcar que el conflicto representado no tiene que resolverse positivamente, sino que buscamos la dramatización de conflictos mal gestionados para poder trabajar sobre la mejor resolución de forma grupal. Antes y después de la representación de la escena se inicia un proceso de reflexión.

- Asignación de roles emocionales: personaje disruptivo, empático, pasivo, implicado, mediador, víctima, agresor, etc.

- Identificación de actitudes y comportamientos: positivos, negativos, pasivos y neutrales.

- Reparto de papeles atendiendo a las características de los miembros del grupo.

\section{Fase de puesta en Escena}

3.1 Representación ordenada de las escenas creadas

- Preparación del espacio.

- Cuidado del ambiente de aprendizaje: potenciar el respeto e interés para favorecer el diálogo tras la puesta en escena.

\subsection{Interpretación de la escena por los grupos de espectadores}

- El docente como generador de diálogo constructivo.

- Posibilidad de registrar las contribuciones en fichas elaboradas previamente, notas o plantillas de evaluación.

- Identificación de conductas negativas, roles, actitudes características, emociones, sentimientos y consecuencias.

\section{Fase de Evaluación}

4.1 Coevaluación, autoevaluación y evaluación docente

- De las tareas realizadas.

- Del contenido trabajado.

- Evaluación contextualizada desde la realidad cercana del alumnado que potencie la reflexión.

\section{Planteamiento metodológico de la Propuesta}

Implementar en las aulas de Educación Primaria esta Propuesta de educación emocional requiere que los docentes piensen y reflexionen sobre cuestiones tales como: la 
organización del alumnado, el ambiente de aprendizaje, la gestión del aula, los materiales y recursos, los procesos de observación/evaluación, etc.

La dinámica de clase que proponemos es eminentemente práctica desde el desarrollo de asambleas, grupos de discusión, diferentes técnicas de grupo y role playing. Sin embargo, en momentos será necesario introducir contenidos con mayor carácter teórico, sobre todo en lo relativo a identificación y definición de las emociones. El dominio de los contenidos de la Propuesta requiere, además, un trabajo individual por parte del docente en educación emocional y recursos dramáticos que le permita desarrollarlo de manera óptima. Esto conllevará la lectura de documentos, el intercambio de experiencias, la representación mental previa del proceso que facilite el reconocimiento de necesidades y todas aquellas estrategias que faciliten la concreción del plan y la atención a las necesidades del grupoclase.

Un ejemplo de recurso a utilizar en la Propuesta y que puede enfocarse desde muchas perspectivas es el modelo de hoja de trabajo que presentamos a continuación (Bisquerra, 2008: 205). La hoja puede tratarse como mejor corresponda: puede ser cumplimentada individualmente, compartida, verbalizada, etc. Y del mismo modo, puede utilizarse para objetivos diferentes como fomentar el diálogo, comenzar una asamblea, presentar un informe, entre otros. Además, las preguntas ofrecen al alumnado la posibilidad de reconstruir sus saberes, de relacionar lo que ocurre en clase con el tema que están trabajando.

Tabla 1.

Hoja de trabajo "Siento y pienso" (Bisquerra, 2008: 205).

\begin{tabular}{|l|l|}
\hline Cuando... & \\
\hline Me siento... & \\
\hline Y pienso... & \\
\hline $\begin{array}{l}\text { Como consecuencia... } \\
\text { (¿Qué hago?) }\end{array}$ & \\
\hline
\end{tabular}

Asumimos con Lomas (1996) que el aula es un contexto complejo e imprevisible en el que se experimenta, se cometen errores y se buscan soluciones. Asimismo, Loughlin y Suina (1990: 21-22) señalan que "el entorno de aprendizaje puede ser un poderoso instrumento docente a disposición del profesor o puede constituir una influencia no dirigida ni reconocida, tanto sobre las conductas de los profesores, como sobre la de los niños". Dominar el ambiente de aprendizaje significa incorporar explícitamente dinámicas, tareas, contenidos, materiales y organización de espacios y tiempos adecuados al planteamiento docente que se persigue.

\section{Discusión y conclusiones}

Queremos señalar aquí la importancia de un proceso de educación emocional fundamentado en las necesidades del alumnado de Educación Primaria y que gestione la complejidad de los diferentes contextos educativos. 
La escuela del siglo XXI tiene que asumir la responsabilidad de educar las emociones de los niños tanto o más que la familia. Los educadores han de ser los principales líderes emocionales del alumnado. La puesta en práctica de programas de educación emocional requiere una formación previa del profesorado. De ahí la importancia de incluir el desarrollo de competencias emocionales de forma intencional y sistemática en los Planes y Programas de Formación Inicial y Permanente del Profesorado.

Consideramos que la formación inicial debería dotar de un bagaje sólido en materia de emociones y fundamentalmente de competencias emocionales. Esto permitirá a los docentes comprender la relevancia de las emociones en la educación y en la vida diaria para tomar conciencia de las propias emociones y de las emociones de los demás, en particular del alumnado, en las interacciones sociales. Se trata de aprender estrategias que nos faciliten el afrontar la tarea educativa en toda su complejidad.

La propuesta de trabajo que presentamos es un tema abierto y será la implementación en las aulas y el análisis cualitativo de las diferentes situaciones vividas las que nos proporcionen evidencias para analizar. El saber comunicarse y reconocer emociones propias/ajenas, son habilidades imprescindibles para que los alumnos maduren progresivamente y así alcancen una solvencia adecuada para integrarse en la sociedad y ser felices. Nosotros podemos darles esa oportunidad.

\section{Referencias}

Bisquerra, R. (2012). Diversidad y escuela inclusiva desde la educación emocional. Diversidad, calidad y equidad educativas, 1-23.

Bisquerra, R. (2008). Educación para la ciudadanía y convivencia, el enfoque de la educación emocional. WK Educación.

Bisquerra, R. (2003). Educación emocional y competencias básicas para la vida. Revista de investigación educativa, 21(1), 7-43.

Bisquerra, R. y Pérez, N. (2007). Las competencias emocionales. Educación XXI, 10, 61-82.

Bruner, J. (1998). Realidad mental, mundos posibles. Barcelona: Gedisa.

Freire, P. (1997). Pedagogía del Oprimido. Madrid: Siglo XXI de España Editores.

Gómez, J. (2003). Educación emocional y lenguaje en la escuela. Barcelona: Ediciones Octaedro.

Lomas, C. (1996). La comunicación en el aula. Signos. Teoría y Práctica de la Educación, 17. Centro de Profesores: Gijón.

Loughlin, C. y Suina, J. (1990). El ambiente de aprendizaje. Madrid: Morata.

Mallika, H. (2000). Drama's Ways of Learning. Research in Drama Education, 5 (1), 45-62.

Mato, M. (2006). El baúl mágico. Imaginación y creatividad con niños de 4 a 7 años. Ciudad Real: Ñaque.

Mora, F. (2013). ¿Qué es una emoción? Revistas Científicas del CSIC, Vol 189, No 759.

Navarro, M. R. (2007). Drama, creatividad y aprendizaje vivencial: Algunas aportaciones del drama a la educación emocional. Cuestiones Pedagógicas, 18, 161-172

Núñez, L. y Navarro, M. R. (2007). Dramatización y educación: aspectos teóricos. Teoría de la educación, 19, 225-252. 
Pérez, M. (2004). La dramatización como recurso clave en el proceso de enseñanza y adquisición de las lenguas. Glosas Didácticas, 70-80.

Prado, J. (2011). Didáctica de la Lengua y la Literatura para educar en el siglo XXI. Madrid: La Muralla.

Real Decreto 126/2014 por el que se establece el currículo básico de la Educación Primaria. Madrid: Boletín Oficial del Estado, Número 52.

Rulicki, S., \& Martín, C. (2012). Comunicación no verbal. Cómo la inteligencia emocional se expresa a través de los gestos. Buenos Aires: Ediciones Granica.

UNESCO (2015). Replantear la educación ¿Hacia un bien común mundial? París: UNESCO.

Winston, J. (2000). Drama, Literacy and Moral Education 5-11. London: David Fulton.

Zillmann, D. (1994). Mechanisms of emotional involvement with drama. Poetics, 23, 33-51. 